\title{
Resonant Geometric Phases for Soliton Equations
}

\author{
M.S. Alber \\ Department of Mathematics \\ University of Notre Dame, Notre Dame \\ IN 46556 , USA \\ J.E. Marsden \\ Department of Mathematics \\ University of California, Berkeley \\ CA 94720, USA
}

\section{To the memory of Solomon J. Alber}

\section{Introduction}

Methods of symplectic and complex geometry have recently given important information about the phase-space geometry of nonlinear equations (see, for example, Ercolani, Forest, McLaughlin, and Montgomery [1987], Ercolani [1989], Ercolani and McLaughlin [1991], McLaughlin and Overman [1993]). A few specific examples are as follows. From the point of view of complex geometry, the reality conditions and homoclinic varieties for the sine-Gordon (SG) equation

$$
U_{x x}-U_{t t}=\sin U
$$

were investigated in Ercolani and Forest [1985] and Ercolani [1989]. The modulational Poisson structure for the sine-Gordon system was derived in terms of conformal ingredients, such as differentials on Riemann surfaces and $\theta$-functions, and a link with the Hamiltonian theory was investigated (see Ercolani et. al. [1987]). Geometric singular perturbation theory was discussed in Kovacic and Wiggins [1992] in connection with the resonances of the sine-Gordon equation. In Bishop, McLaughlin and Solerno [1989] a global 2-dimensional system of real action-angle variables was constructed for the sine-Gordon equation, which gives a framework for describing the breather-kink-antikink transition. Then a geometric method of "blowing-up the singularity" was used for completing the phase space in the neighborhood of the singular point of the corresponding dynamical system and for extending it to a Hamiltonian system on this new phase space. (For details about a method of "blowing-up the singularity", see Birnir [1986].) Complex geometry related to

1991 Mathematics Subject Classification. Primary 58F07; Secondary $70 \mathrm{H} 99$.

Supported in part by the Ministry of Colleges and Universities of Ontario and the Natural Sciences and Engineering Research Council of Canada while visiting The Fields Institute. 
quasiperiodic and soliton solutions and singular theta functions was studied in the KdV case in McKean [1977] and Ercolani [1989]. Finally, a topological classification of soliton equations based on the geometry of compact invariant varieties was studied in Ercolani and McLaughlin [1991].

In Alber and Marsden [1992, 1994] we developed a new method for obtaining geometric phase phenomena for soliton equations, including the familiar phase shift of interacting solitons. Traditionally, the phase spaces of integrable systems were viewed as being foliated by invariant tori; however, for soliton phase spaces and to get formulas for geometric phases, we have shown that a foliation by noncompact varieties is essential. The method is based on the introduction of a new complex angle representation on a noncompact invariant variety. (In particular, a 1-soliton solution is identified with the linearized Hamiltonian flow on a logarithmic Riemann surface.) Asymptotic reduction of the $n$-dimensional soliton complex angle representation then yields a description of geometric phases in terms of the monodromy at singularities in the space of parameters.

In the process of investigating these angle representations, we deal in a natural way with the phenomena of geometric phases. Recall that in Berry [1984], a geometric phase factor $\exp (i \gamma)$ was considered for systems that are slowly (adiabatically) transported along closed curves in a space of parameters. In Montgomery [1988] a class of connections was constructed to obtain expressions for the Hannay-Berry phases (these are geometric angle shifts in the classical case) for some integrable problems in terms of the nontrivial holonomy of these connections. (See Berry and Hannay [1988].) Montgomery also gave an example of a phase associated with the presence of singularities in the case of a flat connection. Symmetry and reduction were used in Marsden, Montgomery and Ratiu [1990] to obtain a generalization of geometric phases to the non-integrable case in the form of the holonomy of the Cartan-Hannay-Berry connection. Recently, David and Holm [1992] investigated Hannay-Berry phases for the real Maxwell-Bloch equations on $\mathbf{R}^{3}$.

As was already discussed, we introduced angle representations on noncompact Jacobi varieties and used asymptotic reduction to obtain geometric phases for a pair of commuting soliton Hamiltonian flows. Our method uses the fact that asymptotic reduction leads to the complex splitting of the spectrum of the soliton problem. A connection between the $\theta$-function and the $\tau$-function can also be used to find a link between soliton geometric phases and geometric phases for quasiperiodic solutions (see Alber and Marsden [1992] and Alber [1992]). We note that in the quasiperiodic case, geometric phases are related to the Whitham equations through the phase function.

The goal of the present paper is to introduce a multidimensional generalization of asymptotic reduction given in Alber and Marsden [1992], to use this to obtain a new class of solutions that we call resonant solitons, and to study the corresponding geometric phases. The term "resonant solitons" is used because these solutions correspond to a spectrum with multiple points, and they also represent a dividing solution between two different types of solitons. In this sense, these new solutions are degenerate and, as such, will be considered as singular points in the moduli space of solitons.

As one passes from $n$-soliton solutions to resonant solitons, the spectrum changes continuously, but the invariant variety on which the solitons reside, does not. Thus, one has to use the tools of any perturbative set up with great care. The methods of asymptotic reduction provide these tools. 
These methods give a detailed description of complex phases for the focussing nonlinear Schrödinger, or (f)NLS, equation,

$$
i \dot{Q}+\frac{1}{2} Q^{\prime \prime}+\bar{Q} Q^{2}=0
$$

and the sine-Gordon equation, including phases for the breather-kink-antikink and soliton-separatrix interactions. They also yield a link with the geometric models for resonant solutions, such as umbilic geodesics on quadrics. (See Alber and Marsden [1994].) Our approach also provides a link between solitons and homoclinic orbits of nonlinear systems and should enable one to investigate perturbations of the angle representations of nonlinear systems to detect chaotic behavior in the neighborhood of the homoclinic orbits. In particular, in Alber and Marsden [1994] we show that the Hamiltonian flow associated with homoclinic orbits introduced in Devaney [1978] for the C. Neumann problem coincides with the soliton $x$-flow of the KdV equation. This result leads us naturally to the introduction of homoclinic geometric phases.

We summarize some of the new features found in the present paper using generalizations of the method of asymptotic reduction:

- Our general approach allows one to study the collision, or interaction, of solitons of different type, such as the interaction of a breather and a kink solution, which generates additional phase shifts. The paper also discusses resonant solutions, which, as we have mentioned are degenerate solitons, such as solutions separating breathers and kinks.

- We show that along a 2-dimensional direction in an invariant variety $V$, there is a splitting of the $2 n$-dimensional resonant angle representation into the sum of $n$ copies of 2-dimensional solutions of the same type. This type of behavior was previously observed in the case of soliton solutions along a 1-dimensional direction. Namely, this splitting is the analogue, for resonant solutions, of the familiar asymptotic splitting of an $n$-soliton solution into $n$ individual 1-soliton solutions.

- Finally, we develop the method of discrete asymptotic reduction and demonstrate it using the Toda lattice and new discretizations of the defocusing nonlinear Schrödinger (d)NLS equation and obtain the corresponding phases.

We start by recalling in Section 2 the 1-dimensional method of asymptotic reduction for the associated complex angle representations that yields, among other things, complex geometric phases. In Section 3 we illustrate this method using 2soliton solutions of the focusing (f)NLS equation. We introduce a new kind of complex soliton generated by a collision of two standard solitons, which we call a "ghost" soliton. The word "ghost" is used because one "sees" this part of the solution in the complexification of the system, which perhaps explains why the object was missed up to now. This ghost soliton is necessary for the understanding of the entire interaction and for the asymptotic splitting of the solitons. We also expect that it will play an important role in the perturbation theory of the (f)NLS equation and in applications to nonlinear optics, especially in view of recent efforts to use optical solitons in fiber optics and the importance of temporal and spatial phase shifts in these investigations. (See, for example, Hasegawa and Kodama [1992].) 
The general multi-dimensional method of asymptotic reduction is described in Section 4. This approach is illustrated for the case of resonant solutions of nonlinear equations and is applied to the collision, or interaction, of solitons of different type, such as the interaction of a breather with a kink solution of the sine-Gordon equation as well as the interaction of a soliton with a separatrix. In particular, as is shown in Section 5, such interactions generate new phase shifts, including resonant geometric phases.

Finally, in Section 6 we describe asymptotic reduction for discrete (infinite particle) nonlinear problems that arise from partial differential equations by an "exact" discretization process. We will show that this includes Toda lattices that arise from discretizing the soliton solutions of the defocussing nonlinear Schrödinger equation. (This discretization process is different from standard methods of approximating nonlinear equations described in Toda [1989]; here we sample the solutions of nonlinear equations at discrete spatial points and get an exact solution of the infinite particle Toda lattice equations.) This leads to an introduction of new exponential Hamiltonians, angle representations and geometric phases.

The general theory of continuous and discrete systems that are related to each other through the same Jacobi varieties (level sets in the phase space) was developed by Solomon J. Alber [1989, 1991].

Recently methods of asymptotic reduction were applied to the study of peaked solitons and billiard solutions of a class of nonlinear integrable pde's in Alber, Camassa, Holm and Marsden [1994a]. This class of equations includes a shallow water equation investigated in Camassa and Holm [1993] and Camassa, Holm and Hyman [1993] as well as equations from the Dym hierarchy. The method we use for the shallow water equation also leads to a link between a certain class of solutions of one of the members of the Dym hierarchy and solutions of the Hamiltonian system for an $N$-dimensional elliptic billiard. The details of this link can be found in Alber, Camassa, Holm and Marsden [1994b].

\section{Complex geometric phases}

In Alber and Marsden [1992, 1994] a general approach to geometric phases for soliton equations was described and the (d)NLS and KdV equations were considered in detail.

In what follows, we demonstrate the general method of asymptotic reduction of the complex angle representations for the (f)NLS and sine-Gordon (SG) hierarchies of equations and use methods of complex analysis to yield information about geometric phases. In particular, this method gives a description of a new effect of a residual interaction between 1-soliton solutions after the asymptotic splitting of the $n$-soliton solution of the (f)NLS equation. On the other hand, angle representations for the SG equation give insight into the breather-kink-antikink interaction and describe the effect of such interactions on the transition through the separatrix solution.

One starts with two complex Hamiltonian flows in complex phase space $\mathbf{C}^{2 n}$. The main purpose of asymptotic reduction is to investigate the vector phase function $\varphi$, a $\mathbf{C}^{n}$ valued meromorphic function defined on an invariant variety $V$ consisting of the symmetric product of $n$ copies of a Riemann surface $\Re \subset \mathbf{C}^{2 n}$ associated to the problem. This Riemann surface $\Re$ is determined by the constants of the motion of the particular problem, and was defined for different examples in Alber and 
Marsden [1992]. Asymptotic reduction plays the role of averaging in the sense that it separates the geometric phase from the dynamical phase. Normally the dynamic phase is the frequency times the time (or the integral of the frequency with time (see, for example, Montgomery [1988], page 276). In the case of solitons, the period is infinite, and this is a main difficulty that is overcome by the method of asymptotic reduction. The complex phase function, which stands as an argument in the $\tau$-function, keeps track of the phase information in solitons, even when they are interacting. In this context, the phase shift of solitons is related to the singularity structure of the phase function.

We may regard $V$ as a bundle over a space of parameters $M$. In our case, the parameters consist of spectral parameters $a_{j}$ that will be discussed below. We can choose an arbitrary connection on this bundle. In what follows, we will choose a trivial connection because we are primarily interested in describing geometric phases in terms of monodromy caused by the presence of singularities. In this context, the geometric phase is defined, as in Alber and Marsden [1992], as follows.

Definition 2.1 We define soliton geometric phases as:

$$
\oint_{C} d_{a} \varphi
$$

Here $d_{a}$ denotes the covariant derivative relative to the given connection and $C$ is a closed curve in the space of parameters.

Note that this formula incorporates the possibilities of two sources for geometric phases, namely the anholonomy of a connection, and the monodromy of a singularity. From the point of view of a connection it is a natural extension of the results obtained for the Cartan-Hannay-Berry connection in the real case using averaging techniques

$$
\text { Hannay's angles }=\oint_{C}\left\langle d_{a} \theta\right\rangle .
$$

For details see Montgomery [1988] and Marsden, Montgomery and Ratiu [1990].

\section{Geometric phases for the (f)NLS equation}

It is known that quasiperiodic invariant tori are degenerate for the NLS equation. These degeneracies are related to the fact that the NLS equation should be considered as a degenerate case of a more general nonlinear problem. (For details see Previato [1985] and M. Alber and S. Alber [1985, 1987].)

This difficulty manifests itself also on the level of searching for solitons. In what follows, we show that the method of asymptotic reduction naturally deals with the degenerate nature of the soliton solutions on the level of invariant varieties.

To obtain multi-solitons of the (f)NLS equation, we first consider quasiperiodic solutions. We start with the spectral polynomial

$$
C(\mu)=\prod_{r=1}^{4 N}\left(\mu-m_{r}\right)
$$


depending parametrically on the spectrum $m_{1}, \ldots, m_{4 N}$. The complex numbers $m_{j}$ are assumed to be distinct. Let

$$
D_{j}=\left(-\sum_{l=1, l \neq j}^{g} \mu_{l}+\frac{1}{2} \sum_{r=1}^{4 N} m_{r}\right) .
$$

Here $g=2 N-1$. Next, consider the following Riemann surface:

$$
\Re_{\text {quasi }}: W^{2}=-C(\mu)
$$

and the variety $V=\left(\Re_{\mathrm{quasi}} \times \cdots \times \Re_{\mathrm{quasi}}\right) / \sigma_{g}$ where $\sigma_{g}$ is the group of permutations of $g$ letters. Finally, let $\epsilon_{j}= \pm 1$. One can choose these arbitrarily at the moment; their significance will be discussed later. In terms of this data, we consider the following two systems of differential equations on $V$ :

$$
\left.\begin{array}{l}
\mu_{j}^{\prime}=\frac{\partial \mu_{j}}{\partial x}=2 i \epsilon_{j} \frac{\sqrt{C\left(\mu_{j}\right)}}{\prod_{l \neq j}\left(\mu_{j}-\mu_{l}\right)} \\
\dot{\mu}_{j}=\frac{\partial \mu_{j}}{\partial t}=2 i \epsilon_{j} D_{j} \frac{\sqrt{C\left(\mu_{j}\right)}}{\prod_{l \neq j}\left(\mu_{j}-\mu_{l}\right)}, \quad j=1, \ldots, g
\end{array}\right\} .
$$

Here each variable $\mu_{j}$ lies on a copy of the Riemann surface $\Re_{\mathrm{quasi}}$.

Each of these equations has first integrals given by:

$$
P_{j}^{2}=-C\left(\mu_{j}\right)=-\prod_{r=1}^{4 N}\left(\mu_{j}-m_{r}\right), \quad j=1, \ldots, g .
$$

The equations may be considered as a Hamiltonian system with conjugate variables $\mu_{j}$ and $P_{j}$.

Now we apply the following limiting process to the spectrum $m_{r}$ of the quasiperiodic problem:

$$
m_{4 k}, m_{4 k-1} \rightarrow a_{k}, m_{4 k-2}, m_{4 k-3} \rightarrow \bar{a}_{k}, \quad k=1, \ldots, N .
$$

Here $N=(g+1) / 2$ and $g$, the genus of $\Re_{\mathrm{quasi}}$, is assumed to be an odd number. (See Previato [1985] and McKean [1977] for details.) This limiting process defines a new polynomial $\tilde{C}$ by $\sqrt{C(\mu)} \rightarrow \tilde{C}(\mu)$ and results in the following limiting differential equations (3.4):

$$
\left.\begin{array}{l}
\frac{\partial \mu_{j}}{\partial x}=2 i \epsilon_{j} \frac{\prod_{r=1}^{N}\left(\mu_{j}-a_{r}\right)\left(\mu_{j}-\bar{a}_{r}\right)}{\prod_{l \neq j}\left(\mu_{j}-\mu_{l}\right)}, \\
\frac{\partial \mu_{j}}{\partial t}=2 i \epsilon_{j} D_{j} \frac{\prod_{r=1}^{N}\left(\mu_{j}-a_{r}\right)\left(\mu_{j}-\bar{a}_{r}\right)}{\prod_{l \neq j}\left(\mu_{j}-\mu_{l}\right)}, j=1, \ldots, g
\end{array}\right\}
$$

describing an $N$-soliton solution of the (f)NLS equation. Here

$$
D_{j}=\left(-\sum_{l=1, l \neq j}^{g} \mu_{l}+\sum_{k=1}^{N}\left(a_{k}+\bar{a}_{k}\right)\right),
$$


and $\epsilon_{j}$ is as before. Note that prior to Alber and Marsden [1992], the limiting process (3.6) was usually applied to the system of first integrals as well. As a result, the soliton Hamiltonian flows were considered on pinched tori in the phase space. We have shown that, instead, by introducing the new system of first integrals

$$
P_{j}=\sum_{k=1}^{N} \log \left(\mu_{j}-a_{k}\right)\left(\mu_{j}-\bar{a}_{k}\right) \quad j=1, \ldots, g,
$$

the system can be realized on a noncompact invariant variety: $\left(\Re_{\text {sol }} \times \cdots \times \Re_{\text {sol }}\right) / \sigma_{g}$, where the Riemann surface $\Re_{\text {sol }}$ is defined by

$$
\Re_{\mathrm{sol}}: W=\sum_{k=1}^{N} \log \left(\mu-a_{k}\right)\left(\mu-\bar{a}_{k}\right) .
$$

In Alber and Marsden [1992] we showed that the system (3.7) together with its new first integrals (3.8) can be realized as a Hamiltonian system with an exponential complex Hamiltonian.

Note that the limiting process described above yields an odd dimensional complex (f)NLS system. In what follows we will demonstrate that this yields an additional complex soliton generated by a collision of the standard solitons.

3.1 2-dimensional soliton solution of (f)NLS equation Before describing the $N$-soliton solutions, we want to give a motivation for our approach by describing the "ghost" soliton generated by a collision of two standard optical solitons.

An analysis of the Riemann surface (3.9) associated with the 2-soliton solution of the (f)NLS equation yields $3 \mu$-variables $\left(\mu_{1}, \mu_{2}, \mu_{3}\right)$. This gives 3 terms in the angle representation $\left(\theta_{1}, \theta_{2}, \theta_{3}\right)$ and demonstrates the existence of the 3 -rd "ghost" soliton. In what follows we use the notation: $a_{1}=x_{1}+i y_{1}$ and $a_{2}=x_{2}+i y_{2}$.

The angle representation that describes the collision of 2 standard solitons of the (f)NLS equation, namely

$$
\begin{aligned}
\theta_{1}= & -\frac{1}{4 y_{1}}\left(\sum_{j=1}^{2} \int_{\mu_{j}^{0}}^{\mu_{j}}\left(\frac{1}{\left(\mu_{j}-a_{1}\right)}-\frac{1}{\left(\mu_{j}-\bar{a}_{1}\right)}\right) d \mu_{j}\right) \\
& +\frac{1}{4 y_{1}}\left(\int_{\mu_{3}^{0}}^{\mu_{3}}\left(\frac{1}{\left(\mu_{3}-a_{1}\right)}-\frac{1}{\left(\mu_{3}-\bar{a}_{1}\right)}\right) d \mu_{3}\right) \\
= & x+v_{1} t
\end{aligned}
$$

where $v_{1}=2 x_{1}$ and

$$
\begin{aligned}
\theta_{2}= & -\frac{1}{4 y_{2}}\left(\sum_{j=1}^{2} \int_{\mu_{j}^{0}}^{\mu_{j}}\left(\frac{1}{\left(\mu_{j}-a_{2}\right)}-\frac{1}{\left(\mu_{j}-\bar{a}_{2}\right)}\right) d \mu_{j}\right) \\
& +\frac{1}{4 y_{2}}\left(\int_{\mu_{3}^{0}}^{\mu_{3}}\left(\frac{1}{\left(\mu_{3}-a_{2}\right)}-\frac{1}{\left(\mu_{3}-\bar{a}_{2}\right)}\right) d \mu_{3}\right) \\
= & x+v_{2} t
\end{aligned}
$$


where $v_{2}=2 x_{2}$, also includes the 3 -rd angle variable, which accounts for the remaining interaction between two initial solitons after asymptotic splitting. The third angle variable yields an additional soliton and can be chosen for $t \rightarrow \infty$ and $t \rightarrow-\infty$ as follows

$$
\begin{aligned}
\theta_{3}^{+}= & -\frac{1}{2\left(y_{1}-y_{2}\right)}\left(\sum_{j=1}^{2} \int_{\mu_{j}^{0}}^{\mu_{j}}\left(\frac{1}{\left(\mu_{j}-a_{1}\right)}-\frac{1}{\left(\mu_{j}-a_{2}\right)}\right) d \mu_{j}\right) \\
& +\frac{1}{2\left(y_{1}-y_{2}\right)}\left(\int_{\mu_{3}^{0}}^{\mu_{3}}\left(\frac{1}{\left(\mu_{3}-a_{1}\right)}-\frac{1}{\left(\mu_{3}-a_{2}\right)}\right) d \mu_{3}\right)=\left(x+v_{3} t\right)+\operatorname{Im}\left(\theta_{3}\right), \\
& v_{3}=\frac{2\left(y_{1} x_{1}-y_{2} x_{2}\right)}{\left(y_{1}-y_{2}\right)}
\end{aligned}
$$

and

$$
\begin{aligned}
\theta_{3}^{-}= & \frac{1}{2\left(y_{1}-y_{2}\right)}\left(\sum_{j=1}^{2} \int_{\mu_{j}^{0}}^{\mu_{j}}\left(\frac{1}{\left(\mu_{j}-\bar{a}_{1}\right)}-\frac{1}{\left(\mu_{j}-\bar{a}_{2}\right)}\right) d \mu_{j}\right) \\
& -\frac{1}{2\left(y_{1}-y_{2}\right)}\left(\int_{\mu_{3}^{0}}^{\mu_{3}}\left(\frac{1}{\left(\mu_{3}-\bar{a}_{1}\right)}-\frac{1}{\left(\mu_{3}-\bar{a}_{2}\right)}\right) d \mu_{3}\right)=\left(x+v_{3} t\right)+\operatorname{Im}\left(\theta_{3}\right), \\
& v_{3}=\frac{2\left(y_{1} x_{1}-y_{2} x_{2}\right)}{\left(y_{1}-y_{2}\right)} .
\end{aligned}
$$

Here $\theta_{3}^{-}=-\bar{\theta}_{3}^{+}$. To illustrate the phase shift procedure, we assume that $\left(x_{2}<x_{1}\right)$ and $\left(0 ; y_{2}<y_{1}\right)$, which implies that $\left(v_{2}<v_{1}\right)$. Asymptotic reduction along $\left(d_{1}=x+v_{1} t\right)$ and $\left(d_{2}=x+v_{2} t\right)$ (described in Alber and Marsden [1992]) yields a splitting of the 2 -soliton angle representation and results in the following phase shifts:

$$
\left.\begin{array}{c}
\left|\Delta_{G} \theta_{1}\right|:=\frac{1}{y_{1}} \log \left|\frac{a_{2}-a_{1}}{a_{2}-\bar{a}_{1}}\right|, \\
\operatorname{Arg} \Delta_{G} \theta_{1}:=2 \arctan \left(\frac{y_{2}-y_{1}}{x_{2}-x_{1}}\right)-2 \arctan \left(\frac{y_{2}+y_{1}}{x_{2}-x_{1}}\right) \\
\left|\Delta_{G} \theta_{2}\right|:=-\frac{1}{y_{2}} \log \left|\frac{a_{1}-a_{2}}{a_{1}-\bar{a}_{2}}\right|, \\
\operatorname{Arg} \Delta_{G} \theta_{2}:=-2 \arctan \left(\frac{y_{2}-y_{1}}{x_{2}-x_{1}}\right)+2 \arctan \left(\frac{y_{2}+y_{1}}{x_{1}-x_{2}}\right) .
\end{array}\right\}
$$

Reduction along the third real direction $\left(d_{3}=x+v_{3} t\right)$ yields an additional phase shift, namely the phase shift of the "ghost" soliton corresponding to $\theta_{3}$ :

$$
\left|\Delta_{G} \theta_{3}\right|=\frac{1}{\left(y_{1}-y_{2}\right)} \log \left|\frac{y_{1}}{y_{2}}\right| \text {. }
$$

We choose $\theta_{3}=\theta_{3}^{+}$as $t \rightarrow \infty$ and $\theta_{3}=\theta_{3}^{-}$as $t \rightarrow-\infty$. 
We believe that these new soliton phase shifts can be detected in an experiment similar to those used to measure temporal phase shifts of the optical solitons (see Friberg, Machida and Yamamoto [1992] and Hasegawa and Kodama [1992]).

Remark 3.1 Note that the condition $v_{1}=v_{2}=v$ (or $x_{1}=x_{2}$ ) yields $v_{3}=v$. In other words, the additional soliton generated by standard solitons moving with identical speeds, does not split from them.

To clarify the link between complex angle representations introduced above and standard formulae for optical solitons from nonlinear optics (see Ablowitz and Segur [1981] and Hasegawa and Kodama [1992]) we describe below the 1-dimensional case; that is, the case $(N=1, g=2 N-1=1)$. There is only one $\mu(x, t)$ variable which satisfies the following system of differential equations

$$
\left.\begin{array}{l}
\frac{\partial \mu}{\partial x}=2 i \varepsilon(\mu-a)(\mu-\bar{a}) \\
\frac{\partial \mu}{\partial t}=2 i \varepsilon(\mu-a)(\mu-\bar{a}) D .
\end{array}\right\}
$$

Here $D=(a+\bar{a})=2 \operatorname{Re}(a)$. In what follows we consider $\varepsilon=1$. The choice of the basic point of the angle representation on a particular sheet of the Riemann surface plays an important role in asymptotic reduction for complex solutions of the (f)NLS equation.

The system (3.16) yields:

$$
-\frac{1}{2 i} \int \frac{d \mu}{(a-\mu)(\mu-\bar{a})}=x+v t, \quad v=2 \operatorname{Re}(a),
$$

or

$$
\frac{1}{4 \operatorname{Im}(a)} \int\left(\frac{1}{(a-\mu)}+\frac{1}{(\mu-\bar{a})}\right) d \mu=x+v t
$$

From the equivalent expression

$$
\log \left(\frac{\mu-\bar{a}}{a-\mu}\right)=4 \operatorname{Im}(a)\left(x+v t+\theta_{o}\right)=\beta
$$

it follows that

$$
\frac{\mu-\bar{a}}{a-\mu}=e^{\beta}, \quad \mu=\frac{\bar{a}+a e^{\beta}}{1+e^{\beta}}=\operatorname{Re}(\alpha)-i \operatorname{Im}(a)\left(\frac{1-e^{\beta}}{1+e^{\beta}}\right) .
$$

Now, the solution $Q(x, t)$ of the (f)NLS equation is related to the $\mu$-variable $\mu(x, t)$ by

$$
U=-2 \mu+2(a+\bar{a})=-2 \mu+4 \operatorname{Re}(a)=i \frac{\partial}{\partial x} \log Q
$$

and therefore, we have

$$
Q=\exp \left(-i \int U d x\right)=\exp \left(2 i \int \mu d x-4 i \operatorname{Re}(a) x\right)
$$


Here

$$
\int \mu d x=\operatorname{Re}(a) x-i \operatorname{Im}(a) \int\left(\frac{1-e^{\beta}}{1+e^{\beta}}\right) d x
$$

and

$$
\begin{aligned}
\int\left(\frac{1-e^{\beta}}{1+e^{\beta}}\right) d x & =\int \frac{d x}{1+e^{\beta}}-\int \frac{e^{\beta} d x}{1+e^{\beta}}=\int \frac{e^{-\beta} d x}{e^{-\beta}+1}-\int \frac{e^{\beta} d x}{1+e^{\beta}} \\
& =-\frac{1}{4 \operatorname{Im}(a)} \log \left(e^{-\beta}+1\right)-\frac{1}{4 \operatorname{Im}(a)} \log \left(1+e^{\beta}\right)+r t+c_{0} \\
& =-\frac{1}{4 \operatorname{Im}(a)} \log \left(\frac{e^{-\frac{\beta}{2}}+e^{\frac{\beta}{2}}}{2}\right)^{2}+\delta t+C_{0}
\end{aligned}
$$

Finally, we obtain the expression

$$
Q=Q^{0} \exp \left(-2 i \operatorname{Re}(a) x+2 i \operatorname{Im}(a) \delta t-i \nu_{0}\right) \operatorname{sech}\left(2 \operatorname{Im}(a)\left(x+v t+\theta_{0}\right)\right) .
$$

After substituting the expression for $Q$ into equation (1.2) we determine the coefficient $\delta$. This results in the formula

$$
l Q=Q^{0} \exp \left(-2 i \operatorname{Re}(a) x+2 i\left((\operatorname{Im}(a))^{2}-(\operatorname{Re}(a))^{2}\right) t-i \nu_{0}\right) \operatorname{sech}\left(2 \operatorname{Im}(a)\left(x+v t+\theta_{0}\right)\right)
$$

which coincides with the expression used in nonlinear optics (see Hasegawa and Kodama [1992]).

We expect that angle representation and new phase shifts described above will play an important role in applications to nonlinear optics, especially in view of recent efforts to use optical solitons in fiber optics and the importance of temporal and spatial phase shifts in these investigations. In particular, angle representations can be used for numerical simulations of the solutions of perturbed nonlinear equations.

$3.2 n$-soliton solutions of the (f)NLS equation First of all, we show that (3.7) gives rise to a new Hamiltonian system in the phase space $\mathbf{C}^{2 g}$.

Theorem 3.2 The systems (3.7) are soliton Hamiltonian systems with Hamiltonians

$$
H_{s}^{s}=2 i \frac{\sum_{j=1}^{g}\left(e^{2 i P_{j}}-\bar{C}\left(\mu_{j}\right)\right)}{\prod_{l \neq j}\left(\mu_{j}-\mu_{l}\right)}
$$

and

$$
H_{s}^{d}=2 i \frac{\sum_{j=1}^{g} D_{j}\left(e^{2 i P_{j}}-\bar{C}\left(\mu_{j}\right)\right)}{\prod_{l \neq j}\left(\mu_{j}-\mu_{l}\right)} .
$$

and with first integrals

$$
P_{j}=\frac{\epsilon_{j}}{2 i} \sum_{r=1}^{N} \log \left(\mu_{j}-a_{r}\right)\left(\mu_{j}-\bar{a}_{r}\right) \quad j=1, \ldots, g,
$$

which determine an invariant variety in the form of a symmetric product

$$
\left(\Re_{\mathrm{sol}} \times \cdots \times \Re_{\mathrm{sol}}\right) / \sigma_{g},
$$


of $n$ copies of the Riemann surface described as follows

$$
\Re_{\mathrm{s} o l}: W=\frac{1}{2 i} \sum_{r=1}^{N} \log \left(\mu-a_{r}\right)\left(\mu-\bar{a}_{r}\right) .
$$

Theorem 3.3 The Hamiltonian systems (3.25) and (3.26) have a system of action-angle variables which splits into $N$ 1-soliton angle variables with corresponding phase functions as $(t \rightarrow \infty)$ or $(t \rightarrow-\infty)$.

\section{Proof}

From the system (3.7), we obtain the following system:

$$
\left.\begin{array}{l}
\frac{\mu_{j}^{\prime}}{\left(\mu_{j}-a_{r}\right)\left(\mu_{j}-\bar{a}_{r}\right)}=\epsilon_{r j} \frac{\prod_{s \neq r}^{N}\left(\mu_{j}-a_{s}\right)\left(\mu_{j}-\bar{a}_{s}\right)}{\prod_{l \neq j}\left(\mu_{j}-\mu_{l}\right)} \\
\frac{\dot{\mu}_{j}}{\left(\mu_{j}-a_{r}\right)\left(\mu_{j}-\bar{a}_{r}\right)}=\epsilon_{r j} D_{j} \frac{\prod_{s \neq r}^{N}\left(\mu_{j}-a_{s}\right)\left(\mu_{j}-\bar{a}_{s}\right)}{\prod_{l \neq j}\left(\mu_{j}-\mu_{l}\right)}, \quad r=1, \ldots, N .
\end{array}\right\}
$$

We take into consideration parameters $\epsilon_{r j}= \pm 1$ inherited from the quasiperiodic problem. They will determine in what follows the choice of the sheet of the Riemann surface in the expression for the $r$-th angle variable. Note that a soliton solution corresponds to a particular choice of the indices. Otherwise, the system (3.29) describes a more general class of solutions.

Summing the above equations (3.29) with respect to $j$, we obtain the first part of the angle representation

$$
\begin{aligned}
\theta_{r}= & -\frac{1}{4 \operatorname{Im}\left(a_{r}\right)} \sum_{j=1}^{N} \int_{\mu_{j}^{o}}^{\mu_{j}}\left(\frac{1}{\left(\mu_{j}-a_{r}\right)}-\frac{1}{\left(\mu_{j}-\bar{a}_{r}\right)}\right) d \mu_{j} \\
& +\frac{1}{4 \operatorname{Im}\left(a_{r}\right)} \sum_{j=1, j \neq k}^{N} \int_{\mu_{j}^{o}}^{\mu_{j}}\left(\frac{1}{\left(\mu_{j+N}-a_{r}\right)}-\frac{1}{\left(\mu_{j+N}-\bar{a}_{r}\right)}\right) d \mu_{j+N} \\
= & x+v_{r} t
\end{aligned}
$$

where $v_{r}=2 \operatorname{Re}\left(a_{r}\right)$ and $r=1, \ldots, N$. Here $1 \leq k \leq N$.

We will consider the real part of the angle representation first. It will be shown in the end of this section that phases for the imaginary part of the angle representation are determined by the phases for the real part.

Now we fix the $k$-th direction, $d_{k}=x+v_{k} t=$ constant, and investigate the behavior of the rest of the angle variables

$$
\theta_{r}=x+\left(v_{r}-v_{k}\right) t+v_{k} t=d_{k}+\left(v_{r}-v_{k}\right) t, r=1, \ldots, N: r \neq k,
$$

as $t \rightarrow \pm \infty$. This yields the following limits for the first $(N-1) \mu_{r}$-variables:

$$
\left.\begin{array}{l}
\text { 1. } t \rightarrow \infty: \mu_{r} \rightarrow a_{r} \text {, for } r<k \text { and } \mu_{r} \rightarrow \bar{a}_{r} \text { for } N \geq r>k \\
\text { 2. } t \rightarrow-\infty: \mu_{r} \rightarrow \bar{a}_{r} \text { for } r<k \text { and } \mu_{r} \rightarrow a_{r} \text { for } N \geq r>k \text {. }
\end{array}\right\}
$$

The important part of asymptotic reduction in the case of (f)NLS equation is that it should be applied to the whole vector space of angle representations which has a 
basis consisting of $g=(2 N-1)$ elements. We choose $\epsilon_{j r}$ in a way described below and determine $(N-1)$ missing elements of the basis in each of the cases $t \rightarrow \infty$ and $t \rightarrow-\infty$, as follows:

1. $(t \rightarrow \infty)$ for $r<k$, let

$$
\begin{aligned}
\theta_{r+N}= & \sum_{j=1}^{N} \int_{\mu_{j}^{o}}^{\mu_{j}}\left(\frac{1}{\left(\mu_{j}-a_{k}\right)}-\frac{1}{\left(\mu_{j}-a_{r}\right)}\right) d \mu_{j} \\
& +\sum_{j=1, j \neq k}^{N} \int_{\mu_{j+N}^{o}}^{\mu_{j+N}}\left(\frac{1}{\left(\mu_{j+N}-a_{k}\right)}-\frac{1}{\left(\mu_{j+N}-a_{r}\right)}\right) d \mu_{j+N} \\
= & 2 i\left(a_{k}-a_{r}\right)\left(x+v_{r+N} t\right)
\end{aligned}
$$

and for $N \geq r>k$, let

$$
\begin{aligned}
\theta_{r+N}= & \sum_{j=1}^{N} \int_{\mu_{j}^{o}}^{\mu_{j}}\left(\frac{1}{\left(\mu_{j}-\bar{a}_{k}\right)}-\frac{1}{\left(\mu_{j}-\bar{a}_{r}\right)}\right) d \mu_{j} \\
& -\sum_{j=1, j \neq k}^{N} \int_{\mu_{j+N}^{o}}^{\mu_{j+N}}\left(\frac{1}{\left(\mu_{j+N}-\bar{a}_{k}\right)}-\frac{1}{\left(\mu_{j+N}-\bar{a}_{r}\right)}\right) d \mu_{j+N} \\
= & 2 i\left(\bar{a}_{k}-\bar{a}_{r}\right)\left(x+v_{r+N} t\right) .
\end{aligned}
$$

Here

$$
\left.\begin{array}{l}
v_{r+N}=a_{k}+a_{r} \text { for } r<k, \\
v_{r+N}=\bar{a}_{k}+\bar{a}_{r} \text { for } r>k .
\end{array}\right\}
$$

2. $(t \rightarrow-\infty)$ for $r<k$, let

$$
\begin{aligned}
\theta_{r+N}= & \sum_{j=1}^{N} \int_{\mu_{j}^{\prime}}^{\mu_{j}}\left(\frac{1}{\left(\mu_{j}-\bar{a}_{k}\right)}-\frac{1}{\left(\mu_{j}-\bar{a}_{r}\right)}\right) d \mu_{j} \\
& +\sum_{j=1, j \neq k}^{N} \int_{\mu_{j+N}^{\prime \prime}}^{\mu_{j+N}}\left(\frac{1}{\left(\mu_{j+N}-\bar{a}_{k}\right)}-\frac{1}{\left(\mu_{j+N}-\bar{a}_{r}\right)}\right) d \mu_{j+N} \\
= & 2 i\left(\bar{a}_{k}-\bar{a}_{r}\right)\left(x+v_{r+N} t\right),
\end{aligned}
$$


and for $N \geq r>k$, let

$$
\begin{aligned}
\theta_{r+N}= & \sum_{j=1}^{N} \int_{\mu_{j}^{o}}^{\mu_{j}}\left(\frac{1}{\left(\mu_{j}-a_{k}\right)}-\frac{1}{\left(\mu_{j}-a_{r}\right)}\right) d \mu_{j} \\
& -\sum_{j=1, j \neq k}^{N} \int_{\mu_{j+N}^{o}}^{\mu_{j+N}}\left(\frac{1}{\left(\mu_{j+N}-a_{k}\right)}-\frac{1}{\left(\mu_{j+N}-a_{r}\right)}\right) d \mu_{j+N} \\
= & 2 i\left(a_{k}-a_{r}\right)\left(x+v_{r+N} t\right) .
\end{aligned}
$$

Here

$$
\left.\begin{array}{c}
v_{r+N}=\bar{a}_{k}+\bar{a}_{r} \text { for } r<k \\
v_{r+N}=a_{k}+a_{r} \text { for } r>k .
\end{array}\right\}
$$

Now we take the real parts of the expressions $(3.30),(3.33),(3.34),(3.36)$ and (3.37) and apply asymptotic reduction along the direction $d_{k}(1 \leq k \leq N)$. This yields the following limits for the remaining $(N-1) \mu_{r}$-variables:

$$
\left.\begin{array}{rl}
\text { 1. } t \rightarrow \infty: \mu_{r+N} \rightarrow \bar{a}_{r}, \text { for } r<k \\
& \text { and } \mu_{r+N} \rightarrow a_{r} \text { for } N \geq r>k \\
\text { 2. } t \rightarrow-\infty: \mu_{r+N} \rightarrow a_{r} \text { for } r<k \\
\quad \text { and } \mu_{r+N} \rightarrow \bar{a}_{r} \text { for } r>k .
\end{array}\right\}
$$

After substituting these values into the expression for the $k$-th angle variable it is transformed into the 1 -soliton angle variable with some acquired complex phase:

$$
\begin{aligned}
\Theta_{k}^{+} & =\frac{1}{4 \operatorname{Im}\left(a_{k}\right)} \int_{\mu_{k}^{o}}^{\mu_{k}}\left(\frac{1}{\left(\mu_{k}-a_{k}\right)}-\frac{1}{\left(\mu_{k}-\bar{a}_{k}\right)}\right) d \mu_{k}+\varphi_{k}^{+} \\
& =x+v_{k} t \\
\Theta_{k}^{-} & =-\frac{1}{4 \operatorname{Im}\left(a_{k}\right)} \int_{\mu_{k}^{o}}^{\mu_{k}}\left(\frac{1}{\left(\mu_{k}-a_{k}\right)}-\frac{1}{\left(\mu_{k}-\bar{a}_{k}\right)}\right) d \mu_{k}+\varphi_{k}^{-} \\
& =x+v_{k} t .
\end{aligned}
$$

Here, basic points of the integrals are taken on different sheets of the Riemann surface.

Now using the phase function and the general definition of a geometric phase given above we calculate a real part of the complex (f)NLS phase.

Corollary 3.4 Consider the difference between $\Theta_{k}^{-}$and $\Theta_{k}^{+}$and let $d_{k} \rightarrow \infty$. This yields a phase generated by the singularities of the initial system of equations: 


$$
\begin{aligned}
\Delta_{G} \Theta_{k} & =-\frac{1}{2 \operatorname{Im}\left(a_{k}\right)} \oint_{L_{k}}\left(\frac{1}{\left(\mu_{k}-a_{k}\right)}-\frac{1}{\left(\mu_{k}-\bar{a}_{k}\right)}\right) d \mu_{k}=\varphi_{k}^{-}-\varphi_{k}^{+} \\
& =\frac{1}{2 \operatorname{Im}\left(a_{k}\right)} \sum_{j=1}^{k-1} \log \left|\frac{a_{j}-\bar{a}_{k}}{a_{j}-a_{k}}\right|-\frac{1}{2 \operatorname{Im}\left(a_{k}\right)} \sum_{j=k+1}^{N} \log \left|\frac{a_{j}-\bar{a}_{k}}{a_{j}-a_{k}}\right|
\end{aligned}
$$

Here, the integral is taken along the cycle $L_{k}$ over the basic cut on the Riemann surface.

The method of asymptotic reduction of the angle representations described above yields the splitting of (3.30) into $(N)$ 1-soliton angle representations with their corresponding phase functions. Therefore, the angle representation (3.30) describes $N$-soliton solutions.

Corollary 3.5 The first part (3.30) of the angle representation is the same for all directions $d_{k}$. The choice of the last $(N-1)$ elements of the representation as well as the choice of the $\epsilon_{r j}$ is an important part of the asymptotic reduction in case of the ( $f$ ) NLS equation. The problem is considered on the linear space of the angle representations. Namely, along with every fixed direction $d_{k}$, we choose a new particular set of angle variables $\left(\theta_{r+N}, r=1, \ldots, N, r \neq k\right)$.

To deal with the limiting imaginary part of (3.30), we use the formulae connecting the solution $Q$ of the (f)NLS to the function $U$ :

$$
U=i \frac{\partial}{\partial x} \log Q, \quad U=-2 \sum_{j=1}^{g} \mu_{j}+2 \sum_{k=1}^{N}\left(a_{k}+\bar{a}_{k}\right) .
$$

Integrating this expression, we obtain an arbitrary constant in the phase of the exponent. We put this constant equal to the argument part of the phase corresponding to (3.41). (See the 1-dimensional case discussed in Section 3.1.)

The angle representations introduced above demonstrate the existence of the residual interaction between solitons after the asymptotic splitting of $N$-soliton solutions of the (f)NLS. This residual interaction is a manifestation of the degeneracy of the invariant varieties in the phase space of this soliton problem. It generates the ghost solitons described in previous section.

\section{Asymptotic reduction and sine-Gordon geometric phases}

Another important problem that can be investigated by the method of complex angle representations is the description of the interaction between different types of soliton solutions of the same nonlinear equation. To demonstrate this, we will investigate the breather-kink-antikink interaction and the effect of this interaction on the separatrix of the sine-Gordon equation and corresponding geometric phases. In Bishop, McLaughlin and Solerno [1989], 2-dimensional real angle variables were introduced to describe a transformation of a breather into a kink-antikink solution through a separatrix. In the present paper, we are investigating an interaction between different types of soliton solutions of the sine-Gordon equation and their effect on the transition through an $n$-dimensional separatrix.

We consider $\left(N=\left(n_{1}+2 n_{2}\right)\right)$-dimensional complex phase space $\mathbf{C}^{N}$ and investigate general soliton solutions using asymptotic reduction of the combination 
of the $n_{1}$-dimensional breather and $n_{2}$-dimensional kink-antikink angle representations. The general approach yields an interesting new class of phases and describes the transition through separatrix solution in the $n$-dimensional case.

To clarify the link with algebraic geometry we begin with the quasiperiodic solutions. (We will be using notations introduced in the previous section for (f)NLS equation.) In the case of the sine-Gordon equation, the associated quasiperiodic system has the form

$$
\begin{gathered}
\frac{\partial \mu_{j}}{\partial x}=2\left(1+G_{N-1}\left(\mu_{j}\right)\right) \frac{\sqrt{-\mu_{j} C\left(\mu_{j}\right)}}{\prod_{i \neq j}\left(\mu_{j}-\mu_{i}\right)} \\
\frac{\partial \mu_{j}}{\partial t}=2\left(1-G_{N-1}\left(\mu_{j}\right)\right) \frac{\sqrt{-\mu_{j} C\left(\mu_{j}\right)}}{\prod_{i \neq j}\left(\mu_{j}-\mu_{i}\right)}, \quad j=1, \ldots, N,
\end{gathered}
$$

defined on the symmetric product of the $n$ copies of the hyperelliptic curve

$$
W^{2}=-\frac{C(\mu)}{\mu}, \quad \text { where } C(\mu)=\prod_{r=1}^{2 N}\left(\mu-m_{r}\right)
$$

is a spectral polynomial. (See M. Alber and S. Alber [1985] and Ercolani, Forest, McLaughlin, and Montgomery [1987].) Here

$$
G_{N-1}(\mu)=\frac{1}{4 \sqrt{\prod_{r=1}^{2 N} m_{r}}} \sum_{l=0}^{N-1} g_{l} \mu^{N-l-1}
$$

with the coefficients from

$$
G(\mu)=\prod_{j=1}^{N}\left(\mu-\mu_{j}\right)=\sum_{r=0}^{N} g_{r} \mu^{N-r} .
$$

Using the different limiting processes:

$$
\begin{aligned}
& m_{2 r}, m_{2 r-1} \rightarrow a_{r}, \text { where } a_{r}=-\alpha_{r}^{2}, r=1, \ldots, n_{1}, \\
& \text { which gives an }\left(n_{1}\right) \text { kink-antikink solution }
\end{aligned}
$$

and

$$
\begin{gathered}
m_{n_{1}+4 r}, m_{n_{1}+4 r-1} \rightarrow h_{r}, m_{n_{1}+4 r-2}, m_{n_{1}+4 r-3} \rightarrow \bar{h}_{r}, r=1, \ldots, n_{2}, \\
\text { which gives an }\left(n_{2}\right) \text { breather solution }
\end{gathered}
$$

applied to different parts of the discrete spectrum $\left(m_{r}\right)$ one can obtain a combination of soliton solutions: breathers and kinks. It leads to the transformation of the basic polynomial: $\sqrt{C(\mu)} \rightarrow \bar{C}(\mu)$, which results in the transformation of the differential systems (4.1) and (4.2). 
4.1 Breather-kink collision and corresponding phase shifts The idea is to use different pieces of the soliton and resonant angle representations to construct representation for the collision in the form of a mosaic defined on the Riemann surface of rather complicated structure. Different pieces are kept together through the same set of $\mu$-variables. Note that the general form of each piece corresponding to a particular type of soliton solution is preserved. What is changed is the dimension of the underlying Riemann surface which is determined by the dimension of the mosaic.

We illustrate the action angle variables produced by our method for the two soliton case of the breather-kink-antikink collision, which corresponds to the following discrete spectrum: $\left(a_{1}, a_{2} ; h, \bar{h}\right)$. Here $a_{1}, a_{2} \in \mathbf{R}$ and $h, \bar{h} \in \mathbf{C}$.

$$
\begin{aligned}
& I_{1}=a_{1}, \quad \theta_{1}=\frac{1}{2} \sum_{j=1}^{4} \int_{\mu_{j}^{o}}^{\mu_{j}} \frac{1}{\left(\mu_{j}-a_{1}\right)} \frac{d \mu_{j}}{\sqrt{-\mu_{j}}}=w_{1} x+v_{1} t \\
& I_{2}=a_{2}, \quad \theta_{2}=\frac{1}{2} \sum_{j=1}^{4} \int_{\mu_{j}^{\circ}}^{\mu_{j}} \frac{1}{\left(\mu_{j}-a_{2}\right)} \frac{d \mu_{j}}{\sqrt{-\mu_{j}}}=w_{2} x+v_{2} t
\end{aligned}
$$

and

$$
\begin{aligned}
& I_{3}=h, \quad \theta_{3}=\frac{1}{2} \sum_{j=1}^{4} \int_{\mu_{j}^{o}}^{\mu_{j}}\left(\frac{1}{\left(\mu_{j}-h\right)} \frac{d \mu_{j}}{\sqrt{-\mu_{j}}}=w_{3} x+v_{3} t\right. \\
& I_{4}=\bar{h}, \quad \theta_{4}=\frac{1}{2} \sum_{j=1}^{4} \int_{\mu_{j}^{o}}^{\mu_{j}} \frac{1}{\left(\mu_{j}-\bar{h}\right)} \frac{d \mu_{j}}{\sqrt{-\mu_{j}}}=\bar{w}_{3} x+\bar{v}_{3} t .
\end{aligned}
$$

Here

$$
w_{r}=\left(1-\frac{1}{4 a_{r}}\right), v_{r}=\left(1+\frac{1}{4 a_{r}}\right) r=1,2
$$

and

$$
w_{3}=\left(1-\frac{1}{4 h}\right), v_{3}=\left(1+\frac{1}{4 h}\right) .
$$

$4.2 n$-dimensional case The systems obtained from (4.1) and (4.2) as a result of the limiting procedures (4.6) and (4.7) can be checked to be Hamiltonian systems with the Hamiltonians

$$
H_{s}^{s}=\frac{\sum_{j=1}^{n}\left(e^{2 \sqrt{-\mu_{j}} P_{j}}-\bar{C}\left(\mu_{j}\right)\right)\left(1+G_{N-1}\left(\mu_{j}\right)\right)}{\prod_{l \neq j}\left(\mu_{j}-\mu_{l}\right)}
$$

and

$$
H_{s}^{d}=\frac{\sum_{j=1}^{n}\left(e^{2 \sqrt{-\mu_{j}} P_{j}}-\bar{C}\left(\mu_{j}\right)\right)\left(1-G_{N-1}\left(\mu_{j}\right)\right)}{\prod_{l \neq j}\left(\mu_{j}-\mu_{l}\right)} .
$$


These systems have a complete set of first integrals

$$
P_{j}=\frac{\log \bar{C}\left(\mu_{j}\right)}{2 \sqrt{-\mu_{j}}}, \quad j=1, \ldots, N
$$

and a system of action-angle variables. Here $N=n_{1}+2 n_{2}$.

The action-angle variables can be described as follows:

$$
I_{r}=a_{r}, \quad \theta_{r}=-\frac{\partial S}{\partial I_{r}}=\frac{1}{2} \sum_{j=1}^{N} \int_{\mu_{j}^{o}}^{\mu_{j}} \frac{1}{\left(\mu_{j}-a_{r}\right)} \frac{d \mu_{j}}{\sqrt{-\mu_{j}}}=w_{r} x+v_{r} t, \quad r=1, \ldots, n_{1},
$$

and

$$
\begin{gathered}
I_{r+n_{1}}=h_{r}, \quad \theta_{r+n_{1}}=\frac{1}{2} \sum_{j=1}^{N} \int_{\mu_{j}^{o}}^{\mu_{j}}\left(\frac{1}{\left(\mu_{j}-h_{r}\right)} \frac{d \mu_{j}}{\sqrt{-\mu_{j}}}=w_{r} x+v_{r} t, \quad r=1, \ldots, n_{2}\right. \\
I_{r+n_{2}+n_{1}}=\bar{h}_{r}, \quad \theta_{r+n_{2}+n_{1}}=\frac{1}{2} \sum_{j=1}^{N} \int_{\mu_{j}^{o}}^{\mu_{j}} \frac{1}{\left(\mu_{j}-\bar{h}_{r}\right)} \frac{d \mu_{j}}{\sqrt{-\mu_{j}}}=\bar{w}_{r} x+\bar{v}_{r} t \\
r=1, . ., n_{2} .
\end{gathered}
$$

Here

$$
w_{r}=\left(1-\frac{1}{4 a_{r}}\right), v_{r}=\left(1+\frac{1}{4 a_{r}}\right) r=1, \ldots, n_{1}
$$

or

$$
w_{r}=\left(1-\frac{1}{4 h_{r}}\right), v_{r}=\left(1+\frac{1}{4 h_{r}}\right) \quad r=1, \ldots, n_{2} .
$$

Note that the soliton (kink-antikink) angle representation for the sine-Gordon equation is similar to a particular case $\left(n_{1}=2 n\right)$ of the KdV and (d)NLS representation investigated in Alber and Marsden [1992]. Therefore, it remains only to describe in detail the pure breather angle representation.

We change variables as follows

$$
\mu_{j}=-\xi_{j}^{2}, \quad h_{r}=-\chi_{r}^{2}
$$

choose $\epsilon_{r j}$ in a way specified below to find that real part of the angle representation is represented in the form

$$
\begin{aligned}
& \theta_{r}= \frac{2\left|h_{r}\right|^{2}}{\operatorname{Re}\left(\chi_{r}\right)\left(4\left|h_{r}\right|^{2}-\operatorname{Re}\left(h_{r}\right)\right)} \sum_{j=1}^{n_{2}}\left(\log \left|\frac{\xi_{j}-\chi_{r}}{\xi_{j}+\chi_{r}}\right|\right) \mid \begin{array}{l}
\xi_{j} \\
\xi_{j}^{0}
\end{array} \\
&-\frac{2\left|h_{r}\right|^{2}}{\operatorname{Re}\left(\chi_{r}\right)\left(4\left|h_{r}\right|^{2}-\operatorname{Re}\left(h_{r}\right)\right)} \sum_{j=n_{2}+1}^{2 n_{2}}\left(\log \left|\frac{\xi_{j}-\chi_{r}}{\xi_{j}+\chi_{r}}\right|\right) \mid \xi_{j} \\
& \xi_{j}^{0}
\end{aligned}
$$


and

$$
\begin{aligned}
\theta_{r+n_{2}} & =\frac{2\left|h_{r}\right|^{2}}{\operatorname{Re}\left(\chi_{r}\right)\left(4\left|h_{r}\right|^{2}-\operatorname{Re}\left(h_{r}\right)\right)} \sum_{j=1}^{2 n_{2}}\left(\log \left|\frac{\xi_{j}-\bar{\chi}_{r}}{\xi_{j}+\bar{\chi}_{r}}\right|\right) \mid \begin{array}{l}
\xi_{j} \\
\xi_{j}^{0}
\end{array} \\
& =x+\left(\frac{4\left|h_{r}\right|^{2}+\operatorname{Re}\left(h_{r}\right)}{4\left|h_{r}\right|^{2}-\operatorname{Re}\left(h_{r}\right)}\right) t .
\end{aligned}
$$

Now we apply asymptotic reduction to the real parts of the first $n_{2}$ elements of the angle representation. This yields the following distribution of the limiting values of the $\mu$-variables along real directions $d_{k}$ :

$$
d_{k}=x+V_{k} t=x+\left(\frac{4\left|h_{k}\right|^{2}+\operatorname{Re}\left(h_{k}\right)}{4\left|h_{k}\right|^{2}-\operatorname{Re}\left(h_{k}\right)}\right) t
$$

$\left.\begin{array}{l}\text { 1. } t \rightarrow \infty: \mu_{r} \rightarrow \chi_{r}, \text { for } V_{r}<V_{k} \text { and } \mu_{r} \rightarrow \bar{\chi}_{r} \text { for } V_{r}>V_{k} \\ \text { 2. } t \rightarrow-\infty: \mu_{r} \rightarrow \bar{\chi}_{r} \text { for } V_{r}<V_{k} \text { and } \mu_{r} \rightarrow \chi_{r} \text { for } V_{r}>V_{k} .\end{array}\right\}$

Here it is useful to keep in mind that $\mu_{j+n_{2}}=\bar{\mu}_{j}$ and $V_{n_{2}}<\ldots<V_{1}$.

Now we combine angle representations and obtain phases acquired by breathers as a result of interaction with kinks and vice versa. This results in the following phase shift:

$$
\begin{aligned}
\Delta_{G} \Theta_{k} & =\frac{2\left|h_{k}\right|^{2}}{\operatorname{Re}\left(h_{k}\right)\left(4\left|h_{k}\right|^{2}-\operatorname{Re}\left(h_{k}\right)\right)} \sum_{j=1}^{k-1} \log \left|\frac{\left(\chi_{j}-\chi_{k}\right)}{\left(\chi_{j}+\chi_{k}\right)} \frac{\left(\chi_{j}+\bar{\chi}_{k}\right)}{\left(\chi_{j}-\bar{\chi}_{k}\right)}\right| \\
& -\frac{2\left|\chi_{k}\right|^{2}}{\operatorname{Re}\left(h_{k}\right)\left(4\left|h_{k}\right|^{2}-\operatorname{Re}\left(h_{k}\right)\right)} \sum_{j=k+1}^{n_{2}} \log \left|\frac{\left(\chi_{j}-\chi_{k}\right)}{\left(\chi_{j}+\chi_{k}\right)} \frac{\left(\chi_{j}+\bar{\chi}_{k}\right)}{\left(\chi_{j}-\bar{\chi}_{k}\right)}\right| \\
& +\Delta_{G} \Theta_{k}^{\text {breather-kink }}
\end{aligned}
$$

where $\Delta_{G} \Theta_{k}^{\text {breather-kink }}$ is the phase acquired by the breather after interaction with the kink solution and is given by the following expression:

$$
\begin{aligned}
\Delta_{G} \Theta_{k}^{\text {breather-kink }} & =\frac{2\left|h_{k}\right|^{2}}{\operatorname{Re}\left(h_{k}\right)\left(4\left|h_{k}\right|^{2}-\operatorname{Re}\left(h_{k}\right)\right)} \sum_{j=1 .\left(v_{j} / w_{j}\right)<V_{k}}^{n_{2}} \log \left|\frac{\left(\alpha_{j}-\chi_{k}\right)}{\left(\alpha_{j}+\chi_{k}\right)} \frac{\left(\alpha_{j}+\bar{\chi}_{k}\right)}{\left(\alpha_{j}-\bar{\chi}_{k}\right)}\right| \\
& -\frac{2\left|\chi_{k}\right|^{2}}{\operatorname{Re}\left(h_{k}\right)\left(4\left|h_{k}\right|^{2}-\operatorname{Re}\left(h_{k}\right)\right)} \sum_{j=1 .\left(v_{j} / w_{j}\right)>V_{k}}^{n_{2}} \log \left|\frac{\left(\alpha_{j}-\chi_{k}\right)}{\left(\alpha_{j}+\chi_{k}\right)} \frac{\left(\alpha_{j}+\bar{\chi}_{k}\right)}{\left(\alpha_{j}-\bar{\chi}_{k}\right)}\right|
\end{aligned}
$$

where $a_{j}=-\alpha_{j}^{2}$. These phases depend on the mutual distribution of the elements of the breather and kink parts of the discrete spectrum.

The additional phase that is acquired by the kink along

$$
d_{k}=x+\frac{v_{k}}{w_{k}}
$$


due to interaction with breathers has the form:

$$
\begin{aligned}
\Delta_{G} \Theta_{k}^{\text {kink-breather }} & =\frac{2\left|\alpha_{k}\right|^{2}}{\left.\operatorname{Re}\left(\alpha_{k}\right)\left(4\left|a_{k}\right|^{2}-1\right)\right)} \sum_{j=1, V_{j}<\left(v_{k} / w_{k}\right)}^{n_{1}} \log \left|\frac{\left(\chi_{j}-\alpha_{k}\right)}{\left(\chi_{j}+\alpha_{k}\right)}\right| \\
& -\frac{2\left|\alpha_{k}\right|^{2}}{\left.\operatorname{Re}\left(\alpha_{k}\right)\left(4\left|a_{k}\right|^{2}-1\right)\right)} \sum_{j=1, V_{j}>\left(v_{k} / w_{k}\right)}^{n_{1}} \log \left|\frac{\left(\chi_{j}-\alpha_{k}\right)}{\left(\chi_{j}+\alpha_{k}\right)}\right| .
\end{aligned}
$$

Phases for the imaginary parts of the angle representation are obtained in the form of argument shifts corresponding to the same logarithmic phase function.

In the case of complex geometric phases, the real and imaginary parts of the phase usually have different physical meanings. For example, in the case of a breather solution of the sine-Gordon equation, these two parts are related to two different types of oscillations of the breather; one is a wave phase oscillation and the other is an amplitude oscillation. For the (f)NLS equation, they correspond to temporal and spatial phase shifts of optical solitons.

Above, we considered asymptotic reduction along real "breather" and "kink" directions. Note that complex angle representations obtained in this paper can be also used for integrating complex Hamiltonian flows along complex directions in $x$ and $t$.

\section{Resonant geometric phases}

Below we introduce $2 n$-dimensional angle representations for resonant solutions of the sine-Gordon equation and describe resonant geometric phases. This technique is of course not restricted to the sine-Gordon equation, but can be applied to any soliton equation. Resonant solutions are considered as singular points in the moduli space of solitons and correspond to multiple points of the discrete spectrum.

As we remarked in the introduction, as one passes from $n$ soliton solutions to resonant solitons, the spectrum changes continuously, but the invariant variety on which the solitons reside, does not. Thus, one has to use the tools of any perturbative set up with great care. The methods of asymptotic reduction provide these tools.

We will show that asymptotic reduction of the resonant angle representations along a 2-dimensional direction splits them into the sum of $n$ 2-dimensional resonant solutions. This kind of asymptotic splitting of solitons was previously known only in the case of $n$-soliton solutions splitting into single solitons, and only along a one dimensional direction. The main difference is that in the present case, the 2-dimensional resonant angle representations are irreducible in the sense that they do not split asymptotically.

In particular, this approach yields a detailed description of the complex phases for the (f)NLS and sine-Gordon equations, including phases for the breather-kink and soliton-separatrix interactions.

A resonant solution can be considered as a separatrix between different soliton solutions of the same nonlinear equation. We will demonstrate this idea using the breather-kink-antikink interaction and the transition through a separatrix in the case of the sine-Gordon equation.

Soliton kink-antikink and breather solutions of the sine-Gordon equation are separated by the separatrix solutions obtained as follows. Namely, one takes in 
(4.17) $n_{1}=2 n_{3}$ and considers the following limiting process:

$$
\left(a_{2 k}, a_{2 k-1}\right) \rightarrow b_{k}, \quad k=1, \ldots, n_{3} .
$$

This provides an example of a resonant solution.

The resonant solution (i.e., separatrix solution) in the case of the sine-Gordon equation can be described by the angle representation

$$
\begin{aligned}
& \theta_{r}=\frac{1}{2} \sum_{j=1}^{2 n_{3}} \int_{\mu_{j}^{o}}^{\mu_{j}} \frac{1}{\left(\mu_{j}-b_{r}\right)} \frac{d \mu_{j}}{\sqrt{-\mu_{j}}}=w_{r} x+v_{r} t+\theta_{r}^{0} \\
& \theta_{r+n_{3}}=\frac{1}{2} \sum_{j=1}^{2 n_{3}} \int_{\mu_{j}^{o}}^{\mu_{j}} \frac{1}{\left(\mu_{j}-b_{r}\right)^{2}} \frac{d \mu_{j}}{\sqrt{-\mu_{j}}}=\rho_{r} x+\nu_{r} t+\theta_{r+n_{3}}^{0} \\
& \quad r=1, \ldots, n_{3} .
\end{aligned}
$$

Here

$$
w_{r}=\left(1+\frac{1}{b_{r}}\right), v_{r}=\left(1-\frac{1}{b_{r}}\right), \rho_{r}=-\frac{1}{b_{r}^{2}}, \nu_{r}=\frac{1}{b_{r}^{2}} .
$$

The first $n_{3}$ integrals can be evaluated as before using change of variables

$$
\mu_{j}=-\xi_{j}^{2}, b_{r}=-\beta_{r}^{2}
$$

resulting in the introduction of the logarithmic phase functions. The phase function for the second part of (5.1) can be obtained using the fact that

$$
\begin{aligned}
\theta_{r+n_{3}} & =-\frac{\partial \theta_{r}}{\partial b_{r}}=\frac{1}{2 \beta_{r}} \frac{\partial \theta_{r}}{\partial \beta_{r}} \\
& =\left.\frac{1}{2\left(2 \beta_{r}\right)^{2}} \sum_{j=1}^{2 n_{3}}\left(\frac{1}{\left|\xi_{j}-\beta_{r}\right|}-\frac{1}{\left|\xi_{j}+\beta_{r}\right|}\right)\right|_{\xi_{j}^{0}} ^{\xi_{j}}-\frac{1}{8\left(\beta_{r}\right)^{2}} \theta_{r} \\
& =\theta_{r+n_{3}}^{0}+\frac{1}{b_{r}^{2}}(t-x)
\end{aligned}
$$

We apply asymptotic reduction by investigating the following system, obtained from the resonant angle representation described above,

$$
\begin{gathered}
\theta_{r}=\left.\frac{1}{4 \beta_{r}\left(1-b_{r}\right)} \sum_{j=1}^{2 n_{3}} \epsilon_{r j} \log \left|\frac{\xi_{j}-\beta_{r}}{\xi_{j}+\beta_{r}}\right|\right|_{\xi_{j}^{0}} ^{\xi_{j}}=x+V_{r} t, \quad V_{r}=\frac{b_{r}-1}{b_{r}+1}, \\
\theta_{r+n_{3}}=\left.\frac{1}{8 \beta_{r}^{3}} \sum_{j=1}^{2 n_{3}} \epsilon_{r j}\left(\frac{1}{\left|\xi_{j}-\beta_{r}\right|}-\frac{1}{\left|\xi_{j}+\beta_{r}\right|}\right)\right|_{\xi_{j}^{0}} ^{\xi_{j}}=\frac{1}{b_{r}^{2}}(x-t)+\frac{1}{8 \beta_{r}^{2}}\left(x+V_{r} t\right), \\
r=1, \ldots, n_{3} .
\end{gathered}
$$

Remark 5.1 Note that we are dealing with $\left(2 n_{3}\right) \mu_{j}$ variables and only $\left(n_{3}\right)$ action variables $b_{j}$. This means that we consider each pair of variables $\mu_{j}$ and $\mu_{j+n_{3}}$ on the two copies of the same Riemann surface with the branch point $b_{j}$. 
The equations corresponding to $\theta_{r}$ and $\theta_{r+n_{3}}$ from the systems (5.4) and (5.5) determine exponential and rational rates of convergence to $\pm \beta_{r}$ for $\xi_{r}$ and $\xi_{r+n_{3}}$ along directions $d_{r}$ and $d_{r+n_{3}}$ respectively

$$
d_{r}=x+V_{r} t, \quad d_{r+n_{3}}=\frac{1}{b_{r}^{2}}(x-t)+\frac{1}{8 \beta_{r}^{2}}\left(x+V_{r} t\right) .
$$

Now we fix two directions $d_{k}$ and $d_{k+n_{3}}$ and investigate the asymptotic limit of every $\xi_{j}$ for $t \rightarrow \pm \infty$.

As a result of the asymptotic reduction, we obtain a 2-dimensional separatrix solution that consists of one logarithmic and one rational type of angle variable, together with the acquired phase function $\varphi$. The logarithmic angle variable is

$$
\begin{aligned}
\Theta_{k}^{+,-} & =\frac{b_{k}}{2\left(1+b_{k}\right)} \int_{\mu_{k}^{o}}^{\mu_{k}}-\frac{1}{\left(\mu_{k}-b_{k}\right)} \frac{d \mu_{k}}{\sqrt{-\mu_{k}}} \\
& +\frac{b_{k}}{2\left(1+b_{k}\right)} \int_{\mu_{k+n_{3}}^{o}}^{\mu_{k+n_{3}}} \frac{1}{\left(\mu_{k+n_{3}}-b_{k}\right)} \frac{d \mu_{k+n_{3}}}{\sqrt{-\mu_{k+n_{3}}}}-\varphi_{k}^{+,-}=x+V_{k} t,
\end{aligned}
$$

and the rational one is

$$
\begin{aligned}
\Theta_{k+n_{3}}^{+,-} & =\frac{1}{2} \int_{\mu_{k}^{o}}^{\mu_{k}} \frac{1}{\left(\mu_{k}-b_{k}\right)^{2}} \frac{d \mu_{k}}{\sqrt{-\mu_{k}}} \\
& +\frac{1}{2} \int_{\mu_{k+n_{3}}^{o}}^{\mu_{k+n_{3}}} \frac{1}{\left(\mu_{k+n_{3}}-b_{k}\right)^{2}} \frac{d \mu_{k+n_{3}}}{\sqrt{-\mu_{k+n_{3}}}}-\varphi_{k+n_{3}}^{+,-}=\rho_{k} x+\nu_{k} t .
\end{aligned}
$$

This also results in the accumulation of the following resonant phases:

$$
\begin{aligned}
\Delta_{G} \Theta_{k} & =\varphi_{k}^{+}-\varphi_{k}^{-}=\frac{1}{2 \beta_{k}\left(1-b_{k}\right)} \sum_{j=1, V_{j}<V_{k}}^{n_{3}} \log \left|\frac{\beta_{j}-\beta_{k}}{\beta_{j}+\beta_{k}}\right| \\
& -\frac{1}{2 \beta_{k}\left(1-b_{k}\right)} \sum_{j=1, V_{j}>V_{k}}^{n_{3}} \log \left|\frac{\beta_{j}-\beta_{k}}{\beta_{j}+\beta_{k}}\right|, \\
\Delta_{G} \Theta_{k+n_{3}} & =\varphi_{k+n_{3}}^{+}-\varphi_{k+n_{3}}^{-}=\frac{1}{8 \beta_{k}^{3}} \sum_{j=1, V_{j}<V_{k}}^{n_{3}}\left(\frac{1}{\left|\beta_{j}-\beta_{k}\right|}-\frac{1}{\left|\beta_{j}+\beta_{k}\right|}\right) \\
& -\frac{1}{8 \beta_{k}^{3}} \sum_{j=1, V_{j}>V_{k}}^{n_{3}}\left(\frac{1}{\left|\beta_{j}-\beta_{k}\right|}-\frac{1}{\left|\beta_{j}+\beta_{k}\right|}\right) .
\end{aligned}
$$

Remark 5.2 Note that the angle representation can be smoothly transformed from breather case to kink-antikink case. The problem is that the system of action variables is degenerate for the resonant (separatrix) solution considered on the same phase space $\mathbf{C}^{N}$ together with breathers and kinks. Here $N=n_{1}+2 n_{2}+2 n_{3}$.

On the other hand, we have enough angle variables to describe the resonant (separatrix) subspace of the phase space and to calculate corresponding phases. 
Now we consider a combination of all elements of the angle representation and link them to each other through the same set of exactly $N=n_{1}+2 n_{2}+2 n_{3}$ variables $\mu_{j}$. Here, each of the $\mu_{j}$ is on the corresponding Riemann surface.

Remark 5.3 Asymptotic reduction developed in this paper for resonant solutions of nonlinear equations enables one to construct and investigate $n$-dimensional umbilic solitons introduced in Alber and Marsden [1994]. It also yields angle representations for the solitons with quasiperiodic background and for $m$-dimensional complex solutions which describe collision of $m$ solitons moving with the same speed.

\section{Discrete asymptotic reduction and geometric phases}

Finally, we give an example of the application of asymptotic reduction to the Toda lattice regarded as a spatial discretization of the defocussing (d)NLS equation. We will do this on the level of the angle representations for the two problems. To make this link, we will choose directions for the asymtotic reduction that are partially discrete. In fact, we will choose directions appropriate to solitons for (d)NLS, and along these directions, we leave the time variable $t$ continuous, but choose the spatial variable $x$ to be discrete.

In particular, our approach provides angle representations and exponential Hamiltonians for the $N$ soliton solutions of the infinite particle Toda lattice (on the line) and gives a clear understanding of the corresponding geometric phases. We will also show that asymptotic reduction of the $N$-soliton angle representation for the Toda lattice yields a splitting into the sum of 1-dimensional solitons similar to the solitons of "finite density" of the (d)NLS. (For details about a connection between Toda lattices and continuous problems, see Toda [1989] and S. Alber [1989, 1991].)

Theorem 6.1 N-soliton solutions for the infinite particle Toda lattice system are described by the following angle representation

$$
\begin{aligned}
\theta_{k} & =\sum_{j=1}^{N} \int_{\mu_{j}^{0}}^{\mu_{j}} \frac{d \mu_{j, k}}{\left(a_{k}-\mu_{j, k}\right) \sqrt{\left(\mu_{j, k}-b_{1}\right)\left(\mu_{j, k}-b_{2}\right)}}=t+r h_{k}+\theta_{k}^{0}, \\
k & =1, \ldots, N ; \quad N=2 n+1,
\end{aligned}
$$

where ( $r$ ) is a discrete variable. The system (6.1) splits into the sum of 1dimensional angle representations of the same type as $t \rightarrow \pm \infty$.

Proof One first works out the Hamiltonian system and associated angle representation for the quasiperiodic case as one does for the NLS equation, as was described before, or in Alber and Marsden [1992]. Then one passes to the $N$ soliton limit by collapsing the spectrum as before. This leads to a new system of angle representations as stated in the theorem. To study the asymptotic splitting, we prepare the following lemma.

Lemma 6.2 The 1-dimensional angle representation

$$
\theta=\int_{\mu^{0}}^{\mu} \frac{d \mu}{(a-\mu) \sqrt{\left(\mu-b_{1}\right)\left(\mu_{k}-b_{2}\right)}}=t+r h+\theta^{0}
$$


corresponds to a class of solutions of the infinite particle Toda lattice, which includes the 1-soliton solutions.

Proof We introduce new variables

$$
\xi^{2}=\frac{\mu-b_{1}}{\mu-b_{2}}, \quad \mu=\frac{\xi^{2} b_{2}-b_{1}}{\xi^{2}-1} .
$$

This results in the following form of $\theta$ :

$$
\theta=\frac{1}{\rho} \log \left|\frac{\xi-\alpha}{\xi+\alpha}\right|=t+r h+\theta^{0}, \quad r \in Z,
$$

where

$$
\alpha^{2}=-\frac{b_{1}-a}{a-b_{2}}, \quad \rho=\sqrt{\left(a-b_{1}\right)\left(a-b_{2}\right)}
$$

From this it follows that

$$
\mu=b_{2}+\frac{\left(b_{2}-b_{1}\right)}{2}\left(\frac{(1-\beta)}{\alpha(1+\beta)-(1-\beta)}-\frac{(1-\beta)}{\alpha(1+\beta)+(1-\beta)}\right),
$$

where

$$
\beta=\exp \left(\rho \theta^{0}+\rho t+\rho r h\right)=\exp (\rho \zeta)
$$

This yields the solution $(q)$ of the Toda lattice in the form

$$
q=C_{0}+\log \left(\frac{1+e^{\nu} e^{\rho \zeta}}{1+e^{-\nu} e^{\rho \zeta}}\right), \quad \nu=\log \left(\frac{\alpha+1}{\alpha-1}\right),
$$

which is the Toda 1-soliton solution. The proof of the splitting is similar to the continuous case. The main difference between the discrete and continuous cases is the description of a "fixed direction" $D_{k}$. Note that we are considering Toda flow (which is linearized in terms of the angle representation) as an "exact" spatial discretization of the soliton flow of the (d)NLS equation on the noncompact invariant variety described in Alber and Marsden [1992]. Therefore, we consider asymptotic reduction for the infinite particle Toda lattice along a spatial discretization of the continuous direction $d_{k}$ used before for the (d)NLS equation. In particular, this yields discrete geometric phases.

Corollary 6.3 The 1-dimensional angle representation in the above lemma corresponding to the case when $b_{1}=b_{2}=b$, namely,

$$
\theta=\int_{\mu^{0}}^{\mu} \frac{d \mu}{(a-\mu)(\mu-b)}=t+r h+\theta^{0}=\frac{\beta}{a-b}
$$

yields an expression for $q$, namely

$$
q=b t+\log \left(1+e^{\beta}\right)+C_{0}
$$

which is similar to a "dark" soliton of the (d)NLS equation. 
Angle representations of this type belong to the same class as action-angle variables first introduced in Flaschka and McLaughlin [1976].

In a forthcoming paper we will describe new types of discretizations of the soliton problem for the (f)NLS equation which can be investigated using the method of this section. Soliton solutions of these new discrete systems can be considered as an approximation (similar to infinite particle Toda lattice models) for optical solitons.

Lastly, angle representations obtained in Alber and Marsden [1994] for homoclinic orbits of the $\mathrm{C}$. Neumann problem (extended to the discrete case by the method of this section) can be used to describe corresponding homoclinic orbits of the stationary Heisenberg chain with classical spins. (For details concerning a connection between discrete version of the C. Neumann problem and the Heisenberg chain see Moser and Veselov [1991]).

Acknowledgements. Mark Alber thanks The Fields Institute for its kind hospitality during two visits in 1993. We also thank Dave McLaughlin for several helpful suggestions.

\section{References}

Ablowitz, M.J., and Segur, H. [1981] Solitons and the Inverse Scattering Transform, SIAM, Philadelphia.

Adler, M., and Van Moerbeke, P. [1980] Completely integrable systems Kac-Moody Lie algebras and curves, Adv. in Math., 38, 267-317.

Alber, M.S. [1992] Complex Geometric Asymptotics, Geometric Phases and Nonlinear Integrable Systems; in Studies in Math. Phys. 3, North-Holland, Elsevier Science Publishers B.V., Amsterdam, 415-427.

[1991] Hyperbolic Geometric Asymptotics, Asymptotic Analysis, 5, 161-172.

Alber, M.S., and Alber, S.J. [1985] Hamiltonian formalism for finite-zone solutions of integrable equations, C.R. Acad. Sc. Paris, 301, 777-781.

[1987] Hamiltonian formalism for nonlinear Schrödinger equations and sineGordon equations, J. London Math. Soc., 36, 176-192.

Alber, M.S., and Marsden, J.E. [1992] On Geometric Phases for Soliton Equations, Commun. Math. Phys., 149, 217-240.

[1994] Geometric Phases and Monodromy at Singularities, NATO Advanced Study Institute, Series C, (to appear).

Alber, M.S., Camassa, R., Holm, D. and Mardsen, J.E. [1994a] The geometry of peaked solitons and billiard solutions of a class of integrable pde's, Lett. Math. Phys. (to appear).

__ $[1994 \mathrm{~b}]$ On the geometry of umbilic solitons (to appear).

Alber, S.J. [1989] On finite-zone solutions of the relativistic Toda lattices, Lett. Math. Phys., 17, 149 155.

[1991] Associated integrable systems, J. Math. Phys., 32, 916-922.

Berry, M.V. [1984] Quantal phase factors accompanying adiabatic changes, Proc. R. Soc. Lond. A, 392, 4557. 
Berry, M.V., and Hannay, J.H. [1988] Classical non-adiabatic angles, J. Phys. A: Math. Gen., 21, L325-L331.

Birnir, B. [1986] Singularities of the complex Korteweg-de Vries flows, Comm. Pure Appl. Math., 39, 283-305.

Bishop, D.W., McLaughlin, D., and Solerno, M. [1989] Global coordinates for the breather-kink (antikink) sine-Gordon phase space: An explicit separatrix as a possible source of chaos, Phys. Rev. A, 40, 6463-6469.

Camassa, R., and Holm, D.D. [1993] An integrable shallow water equation with peaked solitons, Phys. Rev. Lett., 71, 1661-1664.

Camassa, R., Holm, D.D., and Hyman, J.M. [1993] A new integrable shallow water equation, Adv. in Appl. Mech., (to appear).

David, D., and Holm, D.D. [1992] Multiple Lie-Poisson Structures, Reductions, and Geometric Phases for the Maxwell-Bloch Traveling Wave Equation, J. Nonlinear Sci., 2, 241-262.

Devaney, R. [1978] Transversal homoclinic orbits in an integrable system, Amer. J. Math., 100, 631.

Duistermaat, J.J. [1980] On global Action-Angle Coordinates, Comm. Pure Appl. Math., 23, 687-706.

Ercolani, N. [1989] Generalized Theta functions and homoclinic varieties, Proc. Symp. Pure Appl. Math., 49, 87-100.

Ercolani, N., Forest, M., McLaughlin, D.W., and Montgomery, R. [1987] Hamiltonian structure for the modulation equations of a sine-Gordon wavetrain, Duke Math. Journal, 55, 949-983.

Ercolani, N., and Forest, M. [1985] The Geometry of Real Sine-Gordon Wavetrains, Commun. Math. Phys., 99, 1-49.

Ercolani, N., and McLaughlin, D.W. [1991] Toward a topological classification of integrable PDE's; in Proc. of a Workshop on The Geometry of Hamiltonian Systems (T. Ratiu, ed.), MSRI Publications, 22, Springer-Verlag.

Flaschka, H., and McLaughlin, D.W. [1976] Cannonically Conjugate Variables for the Korteweg-de Vries Equation and the Toda Lattice with Periodic Boundary Conditions, Prog. Theor. Phys., 55, 438-456.

Forest, M.G., and McLaughlin, D.W. [1983] Modulation of Sinh-Gordon and SineGordon Wavetrains, Studies in Appl. Math., 68, 11-59.

Friberg, S.R., Machida, S., and Yamamoto, Y. [1992] Quantum-Nondemolition Measurement of the Photon Number of an Optical Soliton, Phys. Rev. Lett., 69, 3165- 3168 .

Hasegawa, A., and Kodama, Y. [1992] Theoretical foundation of optical-soliton concept in fibers; in Progress in Optics XXX (E. Wolf, ed.), North-Holland, Elsevier Science Publishers B.V., Amsterdam.

Guillemin, V., and Sternberg, S. [1983] The Gelfand-Cetlin system and the quantization of complex Flag Manifolds, J. Funct. Anal., 52, 106-128. 
Kaup, D.J., and Newell, A.C. [1978] Solitons as particles, oscillators, and in slowly changing media: a singular perturbation theory, Proc. R. Soc. London, Ser. A, 361, 413-446.

Kovacic, G., and Wiggins, S. [1992] Orbits homoclinic to resonances, with an application to chaos in model of forced and damped sine-Gordon equation, Physica D., $\mathbf{5 7}, 185$.

Knörrer, H. [1984] Singular fibres of the momentum mapping for integrable Hamiltonian systems, J. Reine u. Ang. Math., 355, 67-107.

Marsden, J.E., Montgomery, R., and Ratiu, T. [1990] Cartan-Hannay-Berry phases and symmetry, Contemporary Mathematics, 97, 279, (1989); see also Mem. AMS, 436.

McKean, H.P. [1979] Integrable Systems and Algebraic Curves, Lecture Notes in Mathematics, Springer-Verlag, Berlin. [1977] Theta functions, solitons, and singular curves; in PDE and Geometry, Proc. of Park City Conference (C.I. Byrnes, ed.).

McLaughlin, D.W., and Overman, E.A. [1993] Whiskered tori for integrable pde's and chaotic behavior in near integrable pde's, Surveys in Appl. Math., 1.

Montgomery, R. [1988] The connection whose holonomy is the classical adiabatic angles of Hannay and Berry and its generalization to the non-integrable case, Commun. Math. Phys., 120, 269-294.

Moser, J. [1980] Various aspects of integrable Hamiltonian systems, Progress in Math., 8, Birkhauser, Boston.

Moser, J., and Veselov, A.P. [1991] Discrete versions of some classical integrable systems and factorization of matrix polynomials, Commun. Math. Phys., 139, 217-243.

Mumford, D. [1983] Tata Lectures on Theta I and II, Progress in Math., 28 and 43, Birkhauser, Boston.

Previato, E. [1985] Hyperelliptic quasi-periodic and soliton solutions of the nonlinear Schrödinger equation, Duke Math. Journal, 52, 329 -377.

Ruijsenaars, S.N.M. [1988] Action-angle maps and scattering theory for some finitedimensional integrable systems $I$. The pure soliton case, Commun. Math. Phys., 115, 127-165.

Toda, M. [1989] Theory of Nonlinear Lattices, Springer-Verlag, New York.

Venakides, S. [1985] The generation of modulated wavetrains in the solution of the Korteweg-de Vries equation, Comm. Pure and Appl. Math., 38, 883-909.

Weinstein, A. [1990] Connections of Berry and Hannay type for moving Lagrangian submanifolds, Adv. in Math., 82, 133-159. 